\title{
Labial Adhesions: Facts and Fiction
}

Sir,

Labial adhesions (vaginal synechiae) are a common problem encountered in general pediatric but there are a lot of misconceptions regarding the etiology and management. We had a similar case where a case of labial adhesion was misdiagnosed as case of vaginal agenesis. A three-year-old girl was referred by local practitioner with complaints of absent vagina and burning micturition. The child also had dribbling of urine on standing up after micturition. The child was advised, apart from a urine routine urine examination, a urine culture, an ultrasonography of the pelvis and a micturating cystourethrography to rule out genitourinary abnormalities. On local examination the child was found to have adhesion of the labia minora which lead to the complete closure of the introitus and child voided from a small orfice at the posterior aspect of introitus near the posterior fourchette (Fig. 1). The urine culture was sterile. These adhesions were lysed in the out patient department using a blunt artery forceps after local application of $5 \%$ lidocaine ointment and it revealed a normal urethral and vaginal orifices. The child started on a regimen of estrogen cream application for three weeks. The child is asympomatic after three months of follow up with no recurrence.

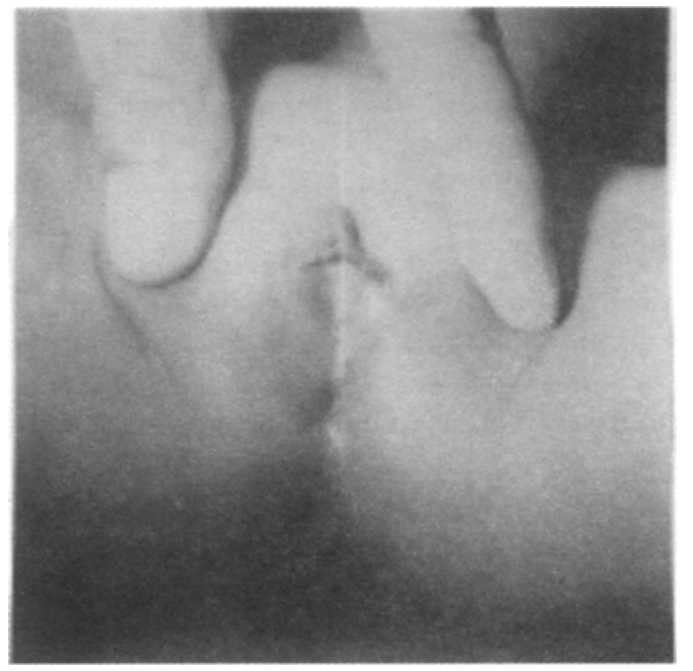

Fig. 1.

Labial adhesions (vaginal synechiae) is a common pediatric gynkechological problem encountered in general pediatric practice and are associated with low estrogen states. ${ }^{1}$ They are commonly misdiagnosed or unnecessarily investigations may be ordered. As many as 1.8 of all patients assessed in a pediatric outpatient clinic may have labial adhesions and present at a peak incidence of 13-23 months but have also been reported to occur much later. ${ }^{2}$ They usually result from chronic inflammation of the vulva and sometimes are associated with sexual abuse. ${ }^{3}$ They have also been reported as a complication of genital herpes and female circumcision. 4,5 They are rarely present at birth, probably due to protective effects of maternal estrogens. Labial adhesions are asymtomatic and are brought by the mother who is anxious about the abnormal anatomy of the urine in the almost completely covered vagina. These patientsmay have dribbling of urine on standing up after micturition and may mimic incontinence. Such urinary stasis may also lead to micturitional disturbance, asymtomatic bacteriuria and to urinary tract infections. ${ }^{6}$

Treatment of labial adhesions consists of estrogen cream application to the labia three times a day for three to four weeks. This usually results in seperation in as many as $50-90 \%$ cases. ${ }^{7.8}$ Patients with dense, fibrous adhesions and girls who have undergone previous surgical separation of the labia are less likely to respond to topical estrogen therapy. ${ }^{8}$ Failure of separation, patients with severe adhesions or patients presenting with urinary tract infections should be managed by manual lysis using a surgical probe under topical anesthesia. Surgical adhesionolysis under general anesthesia may be required in about $5-10 \%$ of cases. ${ }^{8}$ It is imperative to advise local estrogen cream applications after manual or surgical lysis to prevent a recurrence.

\section{Arvind Sinha, Sunita Ojha, Ram Samujh and K.L.N. Rao Department of Pediatric Surgery, Advanced Pediatric Center, PGIMER Chandigarh-160012, India. E-mail:drsinha@vsnl.com}

\section{REFERENCES}

1. Papagiani M, Stanhope R. Labial adhesions in a girl with isolated premature thelarche: the importance of estrogenization. J Pediatric Adolesc Gynecol 2003; 16(1): 31-32.

2. Leung AK, Robson WL, Tay-Uyboco J. The incidence of labial fusion in children. J Pediatr Child Health 1993; 29(3) : 235-236.

3. Kellog ND, Para JM, Menard S. Children with anogenital symptoms and signs referred for sexual abuse evaluations. Arch Pediatr Adolesc Med 1998; 152 (7): 634-641.

4. Mahran M. Medical dangers of female circumcision. IPPF Med Bull 1981; 15(2): 1-3.

5. Herieka E, Dhar J. Labial adhesions following severe primary genital herpes. Sex Transm Infec 2001; 77(1): 75.

6. Leung AK, Robson WL. Labial fusion and urinary tract infection. Child Nephrol Uro 1992; 12 (1): 62-64.

7. Aribarg A. Topical oestrogen therapy for labial adhesions in children. Br / Obstet Gynaecol 1975; 82(5): 424-425

8. Muram D. Treatment of prepubertal girls with labial adhesions. J Pediatr Adolesc Gynecol 1999; 12(2): 67-70. 